\title{
Synthesis of hierarchically porous silica film with controllable surface wettability
}

\author{
Shengyang Tao $\cdot$ Yuchao Wang
}

Received: 22 May 2013/Accepted: 25 November 2013/Published online: 15 March 2014

(C) The Author(s) 2014. This article is published with open access at Springerlink.com

\begin{abstract}
A kind of water-oil separation material was fabricated by depositing the mesoporous microspheres (MS) on filter paper. MS had been prepared via surfactanttemplate method and modified by octyltriethoxysilane. The diameter of microspheres was in the range of 200-800 nm. There were uniform mesopores in the microspheres, and the surface area of microspheres was more than $1,000 \mathrm{~m}^{2} \mathrm{~g}^{-1}$. The thin film, showing both hydrophobicity and lipophilicity, had a high contact angle for water (greater than $130^{\circ}$ ) and a low one for $n$-hexane (about $0^{\circ}$ ). Meanwhile, the film presented excellent separating efficiency between water and oil, which could reach $98.8 \%$. Good selectivity and simple preparation procedure might lead the material to be highly valuable on water-oil separation.
\end{abstract}

Keywords Hierarchically porous - Surfaces - Thin films · Water-oil separation

\section{Background}

Recently, owing to the urgent environmental requirement, there has been an intense increase on the demand for materials able to separate oil spills from water [1-3]. Several separating membranes were reported, such as nano-filtration polymer membranes $[4,5]$, and metal mesh [6-8]. In principle, wettability of solid surfaces is controlled by the chemical composition and the geometrical structure of the surfaces $[9,10]$. Under this condition, a

S. Tao $(\bowtie) \cdot$ Y. Wang

Department of Chemistry, Dalian University of Technology,

Dalian 116024, People's Republic of China

e-mail: taosy@dlut.edu.cn quantity of films with rough and low energy surface have been built and explored well separation property for wateroil. Jiang and Feng [6] reported that the stainless steel mesh coating with Teflon (polytetrafluoroethylene, a low surface-energy material) did well in water-oil separation. Similarly, Yang [7] also prepared a copper mesh with super hydrophobic layer.

Herein, we fabricated a novel hydrophobic hierarchically porous silica film. Via stacking of mesoporous microspheres which are modified by octyltriethoxysilane (OTS), the film has been fabricated showing great performance on the inconsistent liquid mixture separation. With surfactant-template method, mesoporous silica microspheres have been prepared, which is followed by modifying the microsphere with OTS. The modified microspheres can be easily deposited on filter paper. Then, a hierarchically porous film has been formed after filtering and drying. The contact angle for water on this film is greater than $130^{\circ}$, and for $n$-hexane it is $0^{\circ}$ and such novel properties are contributed to the hierarchically porous morphology and the long carbon chain modifier (OTS). The fabrication procedure of our hybrid film is very simple and the materials are of low cost. The film can be used effectively for the separation between water and oil, and may prevail over current membranes as a practical separating material.

\section{Methods}

Ethyl orthosilicate (TEOS), cetyltrimethylammonium bromide (CTAB), ethanol (95\%), ammonia solution (28 wt \%), petroleum ether, toluene, and red dyes were purchased from Beijing Chemical Reagents Company and used without further purification. $N$-octyltriethoxysilane 
Fig. 1 TEM images of MS (a, c) and MS-OTS (b, d); SEM images of the MS-OTS coating film on the filter paper, e large area and $\mathbf{f}$ enlarged image
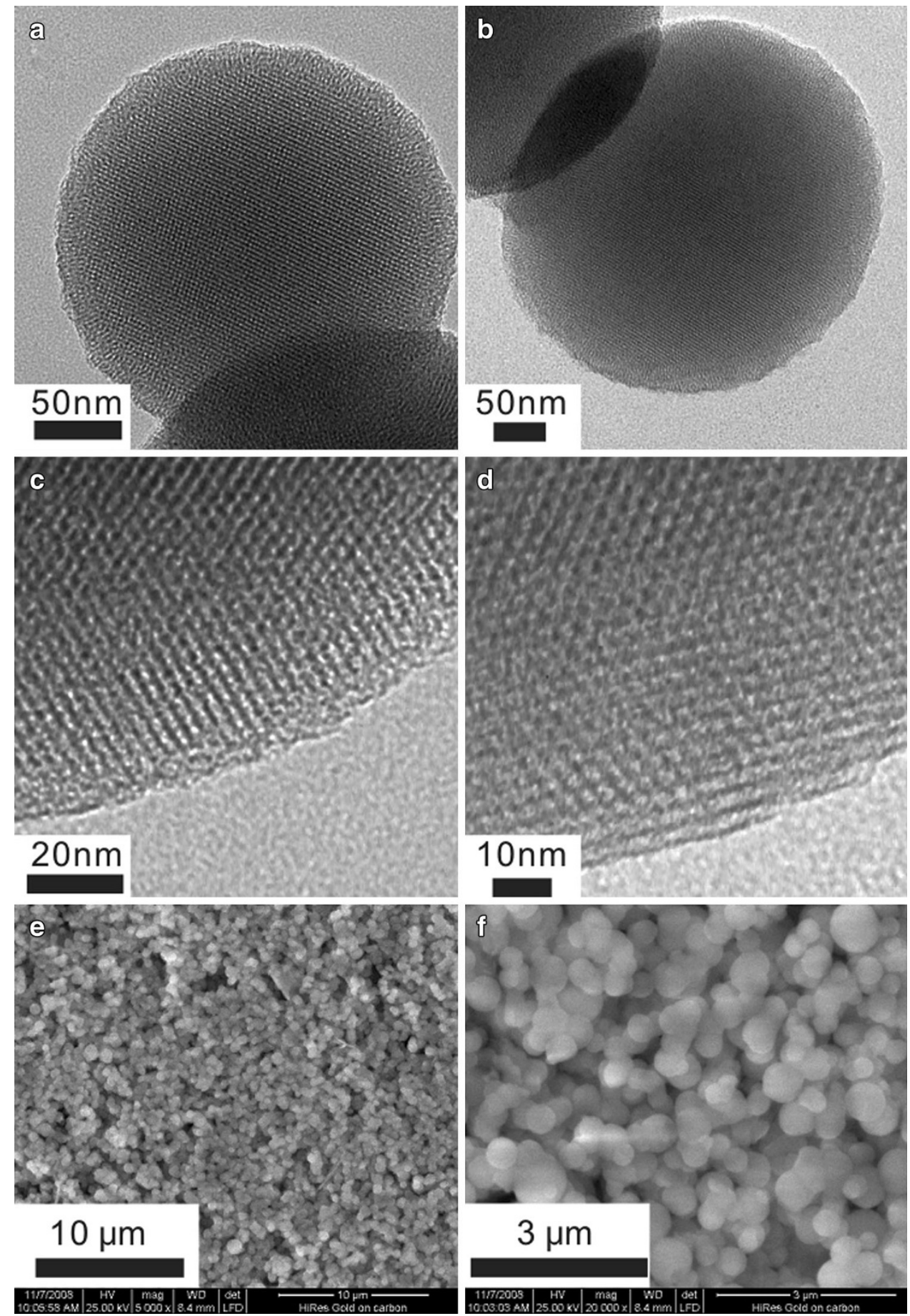

(OTS) was obtained from Aldrich Chemical Co. Inc. The qualitative filter paper (the pore size was about 30-50 $\mu \mathrm{m}$ ) was bought from Dongyang industrial and trading co., LTD (Fushun).

CTAB (1.2 g) was dissolved in deionized water (25 g) and ethanol $(25 \mathrm{~mL})$, and aqueous ammonia $(6 \mathrm{~mL})$ was added. TEOS (1.68 g) was added into the solution after $10 \mathrm{~min}$ stirring. More $2 \mathrm{~h}$ stirring at room temperature, the mesoporous silica microspheres (MS) was recovered by filtration, and calcined at $823 \mathrm{~K}$ for $6 \mathrm{~h}$ to remove the template. The samples were modified by the reaction with OTS. Appropriate amount of MS were allowed to react with modified agents in toluene $(50 \mathrm{~mL})$ at reflux for $12 \mathrm{~h}$. Subsequently, the products were washed with petroleum ether several times, and dried at $373 \mathrm{~K}$ for $12 \mathrm{~h}$. The mesoporous silica modified by OTS (MS-OTS) (0.5 g) was 

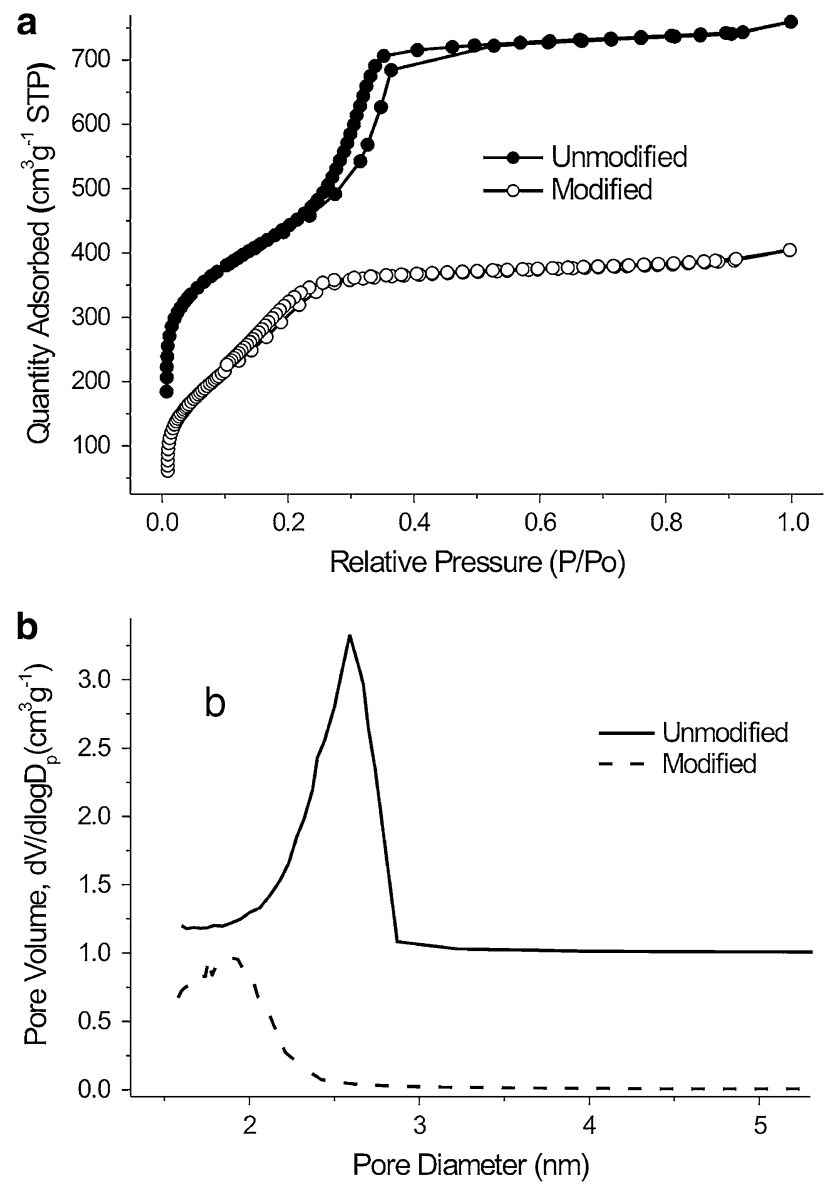

Fig. 2 a $\mathrm{N}_{2}$ adsorption-desorption isotherms and b pore size distribution MS and MS-OTS

dispersed in toluene $(50 \mathrm{~mL})$ and the mixture was deposited on a filter paper by vacuum filtration. Then, a hydrophobic film was gotten after heated at $353 \mathrm{~K}$ for $3 \mathrm{~h}$ in air.

The separating efficiency of hydrophobic film was tested by the oil-water mixture. The simple was prepared by mixing $5 \mathrm{~mL}$ of $n$-hexane into $15 \mathrm{~mL}$ of distilled water. A few drops of red dyes were added for clarity. A certain amount of mixture was filtrated by the as-prepared film. The water, which did not pass the film was gather together and tested for volume. The separating efficiency of water from mixture could be calculated using:

Separating efficiency $(\%)$

$$
=\frac{V_{\text {tested }}(\text { actual volume of water })}{V_{\text {original }}(\text { water volume in mixture })} \times 100 \% .
$$

The scanning electron microscopy (SEM) images were taken with a JEOL JSM-6700F field emission scanning electron microscope (FESEM, $20 \mathrm{kV}$ ). The TEM images were taken on a JEOL-2010 instrument operated at $120 \mathrm{kV}$. The nitrogen adsorption and desorption isotherms were measured at $77 \mathrm{~K}$ using an ASAP 2010 analyzer

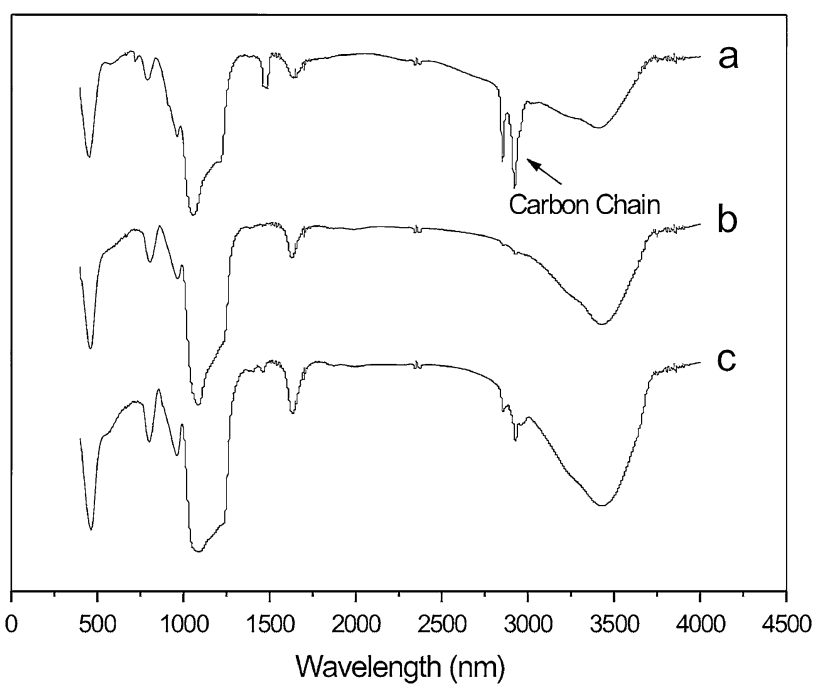

Fig. 3 FTIR spectra of $(a)$ the mesoporous sample, $(b)$ the extracted silica sample, and $(c)$ MS-OTS

(Micromeritics Co. Ltd.). Before measurement, samples were degassed under vacuum at $373 \mathrm{~K}$ for $4 \mathrm{~h}$. A SL200B (Solon Tech. Inc., Ltd. Shanghai) contact angle goniometer was used for static contact angle measurements. The software offered by the instrument manufacturer calculates the contact angles based on the method of the tangent. FT-IR spectra $\left(4,000-500 \mathrm{~cm}^{-1}\right)$ in $\mathrm{KBr}$ were collected on a Nicolet Avatar 360 FT-IR spectrometer by $\mathrm{KBr}$ disc technique.

\section{Results and discussion}

As shown clearly in Fig. 1a, b, the spherical material was with ordered mesopores about $2 \mathrm{~nm}$ in diameter. After modifying the MS, there was no visible change in the microstructure (Fig. 1b) was noted. After depositing the MS-OTS on filter paper, coating film was formed and such film has distinguished by two characteristics, a rough surface and a porous structure. The rough surface had a balllike morphology, and the diameters of the balls are in a random distribution from 200 to $800 \mathrm{~nm}$; a porous structure ensured free passage of air or fluid through the film (shown in Fig. 1c, d).

The specific surface areas and pore size distribution of the prepared samples were determined by nitrogen adsorption-desorption isotherms. As shown in Fig. 2, the isotherms of MS and MS-OTS were both type-IV isotherm under the IUPAC classification. It illuminated that the porous structure would not been destroyed in the modification process. However, the specific areas of MS-OTS decreased from 1,314 to $1,202 \mathrm{~m}^{2} \mathrm{~g}^{-1}$, and pore diameter shrank from 2.5 to $1.9 \mathrm{~nm}$. These changes seemed to be 
Fig. 4 Wettability of filter paper $\mathbf{a}, \mathbf{b}$ coated and $\mathbf{c}$, d uncoated with MS-OTS to (a, c) water or (b, d) $n$-hexane; e the contact angle of water in image (a); $\mathbf{f}$ the separation process of water and oil mixture
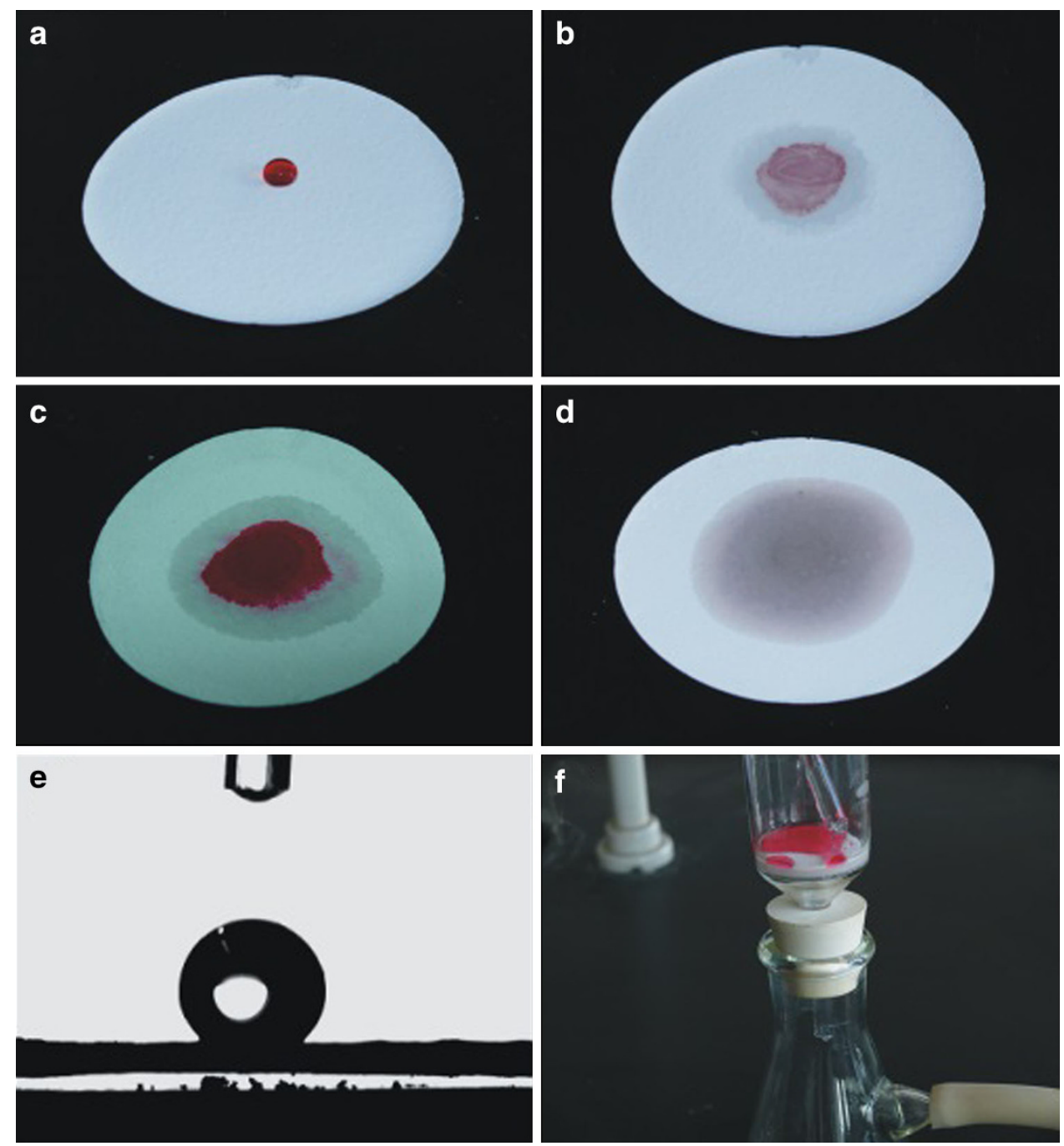

caused by the introduction of long carbon chain which covered the surface of mesopores.

FTIR was also used to prove that the OTS was modified on the surface of MS. As shown in Fig. 3, the characteristic absorption bands of carbon chains were at 2,850 and $2,920 \mathrm{~cm}^{-1}$. Obviously, these bands disappeared after extraction, indicating that $\mathrm{CTAB}$ has been removed. In contrast, besides the absorption bands at 1,066, 952 and $3,000-3,500 \mathrm{~cm}^{-1}$ assigned to $\mathrm{Si}-\mathrm{O}-\mathrm{Si}$ and $\mathrm{Si}-\mathrm{O}-\mathrm{H}$ stretching vibrations, the characteristic adsorption bands of the modified OTS were clearly visible at 2,860 and $2,930 \mathrm{~cm}^{-1}$, which were similar to those of CTAB, but had a small shifting.

There were series of comparisons between the coated and the uncoated filter paper, which were shown in Fig. 4. First, the filter paper coated by MS-OTS worked differently on the area of wettability: hydrophobic (Fig. 4a), yet oleophilic (Fig. 4b). However, as to the uncoated paper, there was no difference on wettability (Fig. 4c, d). was noted. The water CA (contact angle) on this film was 130.8, while the CA of n-hexane was nearly 0 . It meant that hexane could pass through the hydrophobic porous film freely, but water could not. A mixture of hexane and water was used to test the real separation property of the hydrophobic film. After filtration, hexane successfully passed the film without water (Fig. 4e). The separating efficiency of water from mixture was $98.8 \%$. The recycle experiments were shown to measure the stability of the hydrophobic film. The film was simply washed with some petroleum ether and dried at $373 \mathrm{~K}$ for $1 \mathrm{~h}$. Then, it could be totally recyclable. The separating efficiency did not show obvious decrease after 16 cycles (Fig. 5 ).

When compared with the hydrophobic porous film, the modified glass (a sheet glass modified by OTS) has a smaller water contact angle, which is less than $95^{\circ}$. It implies that the surface roughness has played an important role for the high hydrophobicity. The following equation, first derived by Cassie and Baxter, can explain the apparent contact angle on the rough surfaces of hydrophobic porous film [11]. 


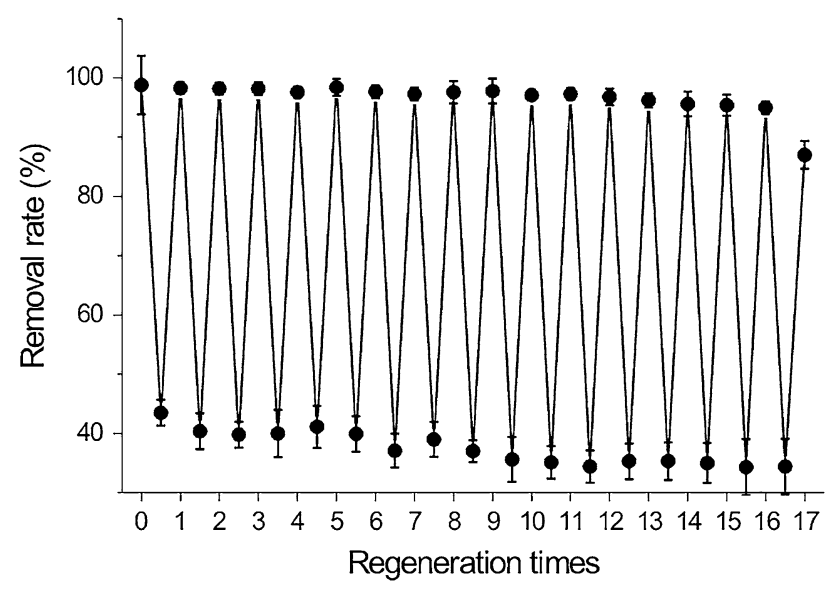

Fig. 5 The regeneration capacity of hydrophobic film with MS-OTS

$\cos \theta_{\mathrm{r}}=f_{1} \cos \theta-f_{2}$,

where $\theta_{\mathrm{r}}$ is the apparent contact angle on the rough surface, $\theta$ is the equilibrium contact angle of the same material, $f_{1}$ is the ratio of surface area of the liquid contacted with the solid to the apparent area and $f_{2}$ is the ratio of surface area of the liquid contacted with air on the rough surface to the apparent area $\left(f_{1}+f_{2}=1\right)$. The $f_{2}$ value of the rough surface is estimated to be 0.64 ( $\theta$ is substituted by the water contact angle of glass). According to the calculated result, it is the fraction of air in the interspaces that is an important factor to get the high hydrophobicity. Obviously, high roughness of porous structure has undoubtedly led to the richness of the fraction of air.

\section{Conclusion}

In summary, a novel hydrophobic separation film has been fabricated. Via adjusting the modifier, the porous silica film exhibits not only hydrophobicity, but also a satisfying property on water-oil separation. In addition, there are also several advantages on preparation such as a simple fabrication procedure and inexpensive raw materials. Furthermore, the discovery is beneficial to design other water-oil separating materials.

Acknowledgments We are genuinely grateful to the National Natural Science Foundation of China (51273030).
Conflict of interest The authors declare that they have no competing interests.

Author's contributions YW participated in the best of materials. ST conceived of the study and synthesized the materials, and drafted the manuscript. Both authors read and approved the final manuscript.

Open Access This article is distributed under the terms of the Creative Commons Attribution License which permits any use, distribution, and reproduction in any medium, provided the original author(s) and the source are credited.

\section{References}

1. Adebajo, M., Frost, R., Kloprogge, J.T., Carmody, O., Kokot, S.: Porous materials for oil spill cleanup: a review of synthesis and absorbing properties. J. Porous Mater. 10, 159 (2003)

2. Yuan, J.K., Liu, X.G., Akbulut, O., Hu, J.Q., Suibm, S.L., Kong, J., Stellacci, F.: Superwetting nanowire membranes for selective absorption. Nat. Nanotechnol. 136, 332 (2008)

3. Zhu, Q., Tao, F., Pan, Q.: Fast and selective removal of oils from water surface via highly hydrophobic core-shell $\mathrm{Fe}_{2} \mathrm{O}_{3} @ \mathrm{C}$ nanoparticles under magnetic field. ACS Appl. Mater. Interf. 2, 3141 (2010)

4. Merkel, T.C., Freeman, B.D., Spontak, R.J., He, Z., Pinnau, I., Meakin, P., Hill, A.J.: Ultrapermeable reverse-selective nanocomposite membranes. Science 296, 519 (2002)

5. Wang, L., Yang, S., Wang, J., Wang, C., Chen, L.: Fabrication of superhydrophobic TPU film for oil-water separation based on electrospinning route. Mater. Lett. 65, 869 (2011)

6. Feng, L., Zhang, Z.Y., Mai, Z.H., Ma, Y.M., Liu, B.Q., Jiang, L., Zhu, D.B.: A super-hydrophobic and super-oleophilic coating mesh film for the separation of oil and water. Angew Chem. Int. Ed. 43, 2012 (2004)

7. Xua, X., Zhang, Z., Yang, J., Zhu, X.: Study of the corrosion resistance and loading capacity of superhydrophobic meshes fabricated by spraying method. Colloids Surf. A 377, 70 (2011)

8. Yang, H., Pi, P.H., Cai, Z.Q., Wen, X.F., Wang, X.B., Cheng, J., Yang, Z.R.: Facile preparation of super-hydrophobic and superoleophilic silica film on stainless steel mesh via sol-gel process. Appl. Surf. Sci. 256, 4095 (2010)

9. Shirtcliffe, N.J., McHale, G., Newton, M.I., Perry, C.C., Roach, P.: Porous materials show superhydrophobic to superhydrophilic switching. Chem. Commun. 25, 3135 (2005)

10. Wang, X.F., Shen, Y.H., Xie, A.J., Qiu, L.G., Li, S.K., Wang, Y.: Novel structure CuI/PANI nanocomposites with bifunctions: superhydrophobicity and photocatalytic activity. J. Mater. Chem. 21, 9641 (2011)

11. Cassie, A.B.D., Baxter, S.: Wettability of porous surfaces. Trans. Faraday Soc. 40, 546 (1944) 